\title{
Diacronie
}

Studi di Storia Contemporanea

$\mathrm{N}^{\circ} 28,4 \mid 2016$

La voce del silenzio

\section{Mauricio Almada Blengio, Crónica de una infamia: el comunicado más vil de la dictadura}

\section{Magdalena Schelotto}

Traduttore: Matteo Tomasoni

\section{OpenEdition}

\section{Journals}

\section{Edizione digitale}

URL: http://journals.openedition.org/diacronie/4462

DOI: $10.4000 /$ diacronie.4462

ISSN: 2038-0925

\section{Editore}

Association culturelle Diacronie

Notizia bibliografica digitale

Magdalena Schelotto, «Mauricio Almada Blengio, Crónica de una infamia: el comunicado más vil de la dictadura ", Diacronie [Online], № 28, 4 | 2016, Messo online il 29 décembre 2016, consultato il 24 septembre 2020. URL : http://journals.openedition.org/diacronie/4462 ; DOI : https://doi.org/10.4000/ diacronie.4462 


\section{Diacronie}

N. 28 | 4|2016 La voce del silenzio: intelligence, spionaggio e conflitto nel XX secolo

$17 /$

\section{PANORAMICA: Cono Sur 2015}

Jacopo BASSI, Carla PEÑALOZA PALMA, Magdalena SCHELOTTO, Ariel SLAVUTSKY, Belen ZAPATA*

SALAZAR VERGARA, Gabriel, La enervante levedad histo rica de la clase polí tica civil (Chile, 19oo-1973), Santiago de Chile, Penguin Random House Grupo Editorial, 2015, 1140 pp.

a cura di Carla PEÑALOZA PALMA

traduzione di Matteo TOMASONI

Senza alcun dubbio, Gabriel Salazar è, oggi come oggi, lo storico vivente più importante del nostro paese e non solamente per la vastità della sua opera, che è ampia e approfondita, ma anche perché è l'autore più conosciuto anche tra coloro che non fanno parte delle cerchie accademiche.

Le sue opinioni - perlopiù controverse - entrano spesso nel dibattito politico. Ma, indipendentemente dal fatto di condividere o meno le sue opinioni, la sua importanza sta nel fatto che abbia allontanato l'idea che gli storici si occupino solamente del passato e che questo non sia d'aiuto per comprendere il presente.

Questo spiega l'impatto de La enervante levedad histórica de la clase política chilena di Gabriel Salazar, che si deve - a mio giudizio - fondamentalmente alla rilevanza del suo autore piuttosto che al suo contenuto.

Quanto detto non significa - in nessun modo - che l'opera non sia interessante. Si tratta di un saggio, tra la storia e la sociologia, di più di mille pagine in cui l'autore ripercorre la storia cilena dall'inizio del XX secolo sino al colpo di Stato, a partire dall'ipotesi che nella storia del Cile i cittadini non abbiano mai esercitato la loro sovranità pienamente e che questa si sia limitata all'esercizio del voto individuale, che non è in alcun modo l'espressione delle loro lotte e speranze.

Salazar afferma nuovamente quello che ha sostenuto in altre opere e in numerose interviste e articoli, ossia che i partiti politici abbiano soppiantato i movimenti sociali, 
impossibilitando ogni relazione tra di essi. I partiti, invece che porsi come interlocutori del movimento sociale prima ancora dello Stato, hanno rappresentato il loro freno ogni qualvolta si sono dedicati all'amministrazione del potere dal momento che non avevano in animo l'idea di trasformare la società.

Per l'autore i partiti, piuttosto, hanno sempre rappresentato una classe endogamica che si è preoccupata solamente della proprio mantenimento al potere, senza che fra di loro si possano operare distinguo nel corso del secolo.

Per questa ragione la sostanza del libro non risiede nella novità, se non nella misura in cui rappresenta la sistematizzazione di un lavoro di anni, in cui l'autore mette in evidenza il ruolo dei movimenti sociali come veri rappresentanti della gente comune.

Si tratta di un lavoro ben documentato e rigoroso che, tuttavia, lascia molti interrogativi: quello forse più importante è come e perché in questo contesto trovò spazio una delle più importanti utopie della sinistra, non solo cliena, ma forse mondiale, e che poté essere fermata solamente con un brutale colpo di Stato che cancellò movimenti sociali e partiti attraverso la repressione.

ÁGUILA, Gabriela, GARAÑo, Santiago, SCATIZZA, Pablo (coord.), Represión estatal y violencia paraestatal en la historia reciente argentina: Nuevos abordajes a 40 años del golpe de Estado, La Plata, FaHCE Universidad Nacional de La Plata, 2016, 483 pp.

a cura di Belen ZAPATA

traduzione di Matteo TOMASONI

L'origine di questo libro risale alla creazione del gruppo chiamato Red de Estudios sobre Represión y Violencia Política (RER), sorto in Argentina nell'anno 2014. Esso sviluppò le sue prime indagini sulla storia recente di questo paese, prestando la sua attenzione in particolar modo al tema della repressione. Basandosi proprio su questo filone di studi, il libro vuole essere un punto di partenza per riunire nuove ipotesi e analisi, ma anche riflessioni fondate sulla ricerca scientifica disponibile. L'opera è divisa in tre parti che sostanzialmente ripartiscono gli elementi tematici dalla riflessione accademica attraverso un ordine preciso: La repressione prima del golpe del 1976, le sue origini e le condizioni che lo permisero; Pratiche, dispositivi ed effetti sociali nel contesto della repressione; Forme e sviluppo della repressione durante la dittatura. Seguendo questo preciso ordine, il libro permette quindi di approfondire con nuovi dati le forme, le pratiche, i dispositivi e gli effetti provocati dalla repressione prima e durante lo sviluppo della dittatura argentina compresa tra il 1976 ed il 1983; 
consente inoltre di riflettere sulla problematica e il dibattito sorto intorno alle periodizzazioni, alle categorie di studio, ma anche alle rappresentazioni sistematiche di questa difficile tematica.

I diversi capitoli che compongono l'opera in questione sono stati arricchiti con minuziose ricostruzioni storiche degli stili di vita, delle istituzioni, delle legislazioni e dei dispositivi creati per la repressione. Troviamo inoltre riflessioni sui procedimenti usati per il sequestro e il rapimento dei bambini (delle famiglie perseguitate), sulla creazione dei servizi di intelligence all'interno di quella che è divenuta nota come "comunità informativa”, sul coordinamento repressivo transnazionale, ecc. La logica a partire dalla quale si struttura il libro è quella di ripensare alla repressione attraverso le particolarità locali e regionali, ma anche attraverso la decentralizzazione operativa creata attraverso zone e aree di controllo militare. Si osserva come la diversità regionale provocò in Argentina un rapido e significativo sviluppo della repressione, in grado di giustificare l'esistenza di un "terrore di Stato" portato a termine dagli enti appositamente creati da quest'ultimo. Su questo aspetto è opportuno ricordare che nel testo si vuole proporre uno studio comparato tra le diverse regioni del paese, così da poter dimostrare l'esistenza di differenti pratiche e metodi di repressione. Lo spettro dell'analisi della repressione diviene invece transnazionale quando si prende in esame l'effetto provocato dall'esilio forzato o le particolarità dell'Operazione Cóndor, in cui venne perpetrato il coordinamento repressivo tra le dittature del Cono Sur più inumano di quest'epoca.

Fra i vari punti di riflessione sviluppati dagli autori, bisogna evidenziare la volontà di riflettere su possibili periodizzazioni della violenza di Stato nel lungo periodo. Vengono proposte nuove ipotesi riguardo al colpo di stato in Argentina del 1955, un momento a partire dal quale iniziarono a consolidarsi le politiche di repressione statale, che ebbero successivamente rilievo nella creazione di un processo di violenza specifico, discontinuo ma comunque unico nel suo genere e nel quale sia civili, sia militari collaborarono nello sviluppo di una logica repressiva poi mantenutasi durante il corso dei decenni. In questo contesto si studia inoltre lo stato di emergenza, il suo funzionamento e gli enti che lo regolarono. Non meno importante è l'analisi della dottrina militare, così come di tutto il corpus dei regolamenti militari nei quali furono sviluppare le specifiche considerazioni sul "nemico interno", ma anche tutte quelle misure di controllo conosciute come regolamenti "anti-sovversivi" imposti dall'Esercito argentino tra il 1955 ed il 1976.

Nell'ultima parte si dà spazio anche ad altri aspetti che sono stati poco o scarsamente studiati sino ad ora e che hanno a che fare con la violenza parastatale o 
con la relazione tra lo Stato e l'esilio, i decreti legge e le altre forme legislative per regolarlo. Questo implica la necessità di studiare la condizione dei detenuti politici e la repressione vissuta negli spazi carcerari attraverso il genere o la sessualità intesi come elementi di controllo ed ulteriore persecuzione all'interno di questi luoghi. Tale studio mette inoltre in luce nuovi aspetti legati al ruolo dei consigli di guerra militari e l'applicazione della repressione politica. Il libro non può quindi non prendere in esame l'analisi dei luoghi dove si sviluppò la repressione, così come la complicità e il grado di responsabilità che ebbero determinati attori civili e i loro rapporti con i militari. Viene sviluppata una riflessione anche sul ruolo esercitato dalla classe lavoratrice e sull'organizzazione di una vera e propria 'resistenza' sorta sin dal periodo predittatoriale, la quale fu causa di ulteriore repressione contro tutti coloro che avevano preso parte a tali manifestazioni o gruppi sindacali. Oltre alle responsabilità civili viene affrontato lo studio sul ruolo svolto da alcuni settori imprenditoriali nell'appoggiare la repressione, partecipando ad essa con mezzi e pratiche basate sulla violenza. Non può mancare, infine, un'ulteriore riflessione sulle responsabilità di altri settori della società, fra i quali spiccano gli appartenenti al settore giudiziario. L'indagine si sviluppa intorno alle continuità e alle rotture interne ai gruppi che presero parte all'amministrazione della giustizia nei diversi regimi dittatoriali dell'Argentina, nell'intento di rompere con la pretesa immagine di asetticità del potere giudiziario, inserendo i suoi operatori - in quanto attori sociali - all'interno di quelle strutture, percorsi, alleanze, gruppi di riferimento e di orientamento ideologico che li caratterizzavano come tali.

\section{SLIPAK, Daniela, Las revistas montoneras, Buenos Aires, Siglo XXI editores, 2015, 269 pp.}

a cura di Ariel SLAVUTSKY

traduzione di Jacopo BASSI

Gli studi sulla violenza politica negli anni Sessanta e Settanta in Argentina si concentrano su due attori principali: lo Stato-nazione e i gruppi politici rivoluzionari.

L'autrice si propone di studiare i processi identitari dei Montoneros utilizzando come fonte le riviste pubblicate fra il 1973 e il 1976. Tra queste troviamo: «Cristianismo y Revolución», benché antecedente alle attività dei Montoneros; tra quelle pubblicate 1973 e il 1974 «El peronista, lucha por la liberación», «La causa Peronista», «El Descamisado», «Evita Montonera» (quest'ultima edita fino al colpo di Stato del 1976); infine le riviste montoneras dissidenti, come «Puro Pueblo» e «Movimiento para la reconstrucción y liberación nacional». 
A partire da queste pubblicazioni l'intento è quello di recuperare i processi di costruzione/invenzione di una storia/tradizione del gruppo sviluppando la ricerca intorno ad alcuni nuclei tematici: l'origine dei Montoneros, il rapporto con Perón e la scelta della violenza rivoluzionaria a partire da cui vengono discusse le idee di deviazione e osservanza della linea.

I capitoli possono essere raggruppati in tre sezioni: il contesto politico generale, la situazione dei Montoneros (dando conto in particolare delle relazioni con le riviste e i loro editori) e, infine, ci si addentra nell'analisi dettagliata delle riviste. Su quest'ultimo punto è importante sottolineare che Slipak non eccede nella quantità di citazioni bibliografiche, il che permette di mantenere un'alta leggibilità del testo.

Per questa ragione il libro si presenta come uno studio interessante, tanto per gli specialisti quanto per i semplici appassionati. Ai primi fornirà un'indagine approfondita che permette di rinverdire vecchie discussioni; ai secondi, invece, permetterà di muovere i primi passi per conoscere la storia recente della repubblica Argentina.

ALMADA BLENGIO, Mauricio, Cró nica de una infamia: el comunicado más vil de la dictadura, Montevideo, Editorial Fin de Siglo, 2015, 152 pp. a cura di Magdalena SCHELOTTO traduzione di Matteo TOMASONI

L'opera in questione, il cui autore è il giornalista uruguayano Mauricio Almada, cerca di approfondire un episodio quasi del tutto sconosciuto della dittatura civilemilitare uruguayana (1973-1985): il sequestro e la tortura di 25 minorenni (14 ragazze e 11 ragazzi) e di una decina di dirigenti comunisti avvenuta nella sede del decimo battaglione della fanteria del dipartimento di Treinta y Tres (regione occidentale del paese) durante l'anno 1975. La ricerca portata avanti dal giornalista ricostruisce e documenta, per filo e per segno, i diciotto giorni trascorsi da queste persone all'interno delle strutture militari.

Almada ha sviluppato la sua indagine prendendo in considerazione anche il contesto internazionale di quest'epoca, sottolineando come l'appoggio degli Stati Uniti ai governi militari latinoamericani fu indubbiamente fondamentale dal momento che il caso uruguayano risultò una sorta di esperimento. L'episodio studiato si inserisce nell'“Anno dell'Orientalità" (Año de la Orientalidad), decretato dalla dittatura nel 1975, che mirava a impiantare un sistema educativo basato sui "buoni modelli di vita" del popolo uruguayano. 
Nonostante fossero stati messi fuorilegge, sia il Partito Comunista dell'Uruguay (PCU) sia l'Unione della Gioventù Comunista (UJC) continuarono la loro militanza, seppur in forma clandestina. Almada documenta l'esistenza di una tortura sistematica alla quale furono sottoposti i giovani militanti della UJC sequestrati dal regime, recuperando alcune testimonianze dirette. Le sessioni dedicate alla tortura si svilupparono nel corso di diversi giorni, ma videro il loro apogeo il 19 aprile, festa nazionale, a causa della visita di colui che firmò il rapporto poi inviato allo Stato Maggiore sull'attività delle UJC nella regione di Treinta y Tres, il comandante della IVa Divisione dell'Esercito, Gregorio Álvarez, futuro presidente del governo.

Il libro analizza inoltre il comunicato del Comando Generale dell'Esercito, firmato dal comandante Julio César Valdora e diffuso attraverso la stampa nazionale, in cui si dava notizia e si giustificava l'operazione intrapresa dal governo militare contro le UJC accusate, oltretutto, di gravi episodi di promiscuità e di essere le promotrici di grandi orgie. Al riguardo, non dobbiamo dimenticare che tali accuse furono utilizzate dal regime proprio per suscitare una riprovazione morale collettiva, molto sentita nell'Uruguay dell'epoca, contro ogni tipo di comportamento sociale di questo tenore.

Non è un caso quindi che lo studio di Almada ripercorra proprio la struttura e la diffusione di questo comunicato, usato come un'arma grazie alla quale amplificare la violenza imposta a questi giovani. Eppure è doveroso ricordare che, all'ombra di questa comunicazione ufficiale, si affiancarono anche una serie di specifici obiettivi militari, fra cui l'autore ricorda la diffusione e legittimità dell'azione armata, così come la pratica della persecuzione o del terrore. La strategia adottata dal regime ebbe un ruolo indubbiamente decisivo nel provocare un danno morale alle vittime della persecuzione militare, che si videro nella maggior parte dei casi obbligate ad abbandonare le loro case e a vivere la loro gioventù come cittadini di terza classe. Tale categoria impediva loro di poter studiare, votare o esercitare qualsiasi diritto di partecipazione alla società civile.

La ricostruzione completa e meticolosa di questi fatti, corroborata a sua volta dalle testimonianze delle vittime e suffragata da documenti storici di diversa tipologia, rende l'opera di Mauricio Almada un lavoro imprescindibile per ricostruire le conseguenze provocate dal comunicato militare, dando finalmente voce a coloro che furono vittime della giustizia militare. La ricerca si conclude con l'analisi della denuncia collettiva che diciannove vittime presentarono nell'anno 2012, ma offre anche una riflessione sul ruolo del Partito Comunista durante e a seguito dei fatti qui analizzati. 
BORGES, Leonardo, El Enigma de Purificación, Montevideo, Fin de Siglo Editorial, 2015, 198 pp.

a cura di Jacopo BASSI

La figura di José Artigas è centrale per la storiografia uruguaiana, soprattutto in considerazione della funzione che svolse per creare una narrazione storica in grado di pacificare e unificare bianchi e colorados nella lotta per l'indipendenza del paese. Malgrado, come detto, il grande interesse storiografico intorno alla figura di uno dei grandi libertadores sudamericani, su Purificación - la città che Artigas volle eleggere a capitale federale - si riscontra un silenzio che somiglia a un vero e proprio oblio storiografico. Il libro esce in occasione dei 200 anni dalla fondazione di Purificación. La città, che contava poco più di un migliaio di abitanti, non aveva esattamente le fattezze di una capitale; qui Artigas promulgò il Reglamento da Tierras e realizzò la prima biblioteca e la prima scuola del nuovo Stato. Purificación, tuttavia nacque come campo di prigionia: il suo nome derivava dalla volontà di purificare i prigionieri politici (i filospagnoli contrari alla Revolución Oriental) attraverso il lavoro forzato. Il libro si sviluppa proprio intorno alla volontà di risolvere alcuni interrogativi storici: quello che verte sulla reale natura dell'insediamento di Purificación (fu un campo militare o il luogo di un insediamento civile?), sulla sua esatta ubicazione, sulla sua natura stessa dell'insediamento (era la sede di un governo o un campo di prigionia per gli avversari della rivoluzione?).

BREZZO, Liliana M., RODRÍGUEZ ALCALÁ, Guido, INSTITUTO DE HISTORIA Y MUSEO MiLITAR (PARAGUAY), La Guerra del Paraguay en primera persona: testimonios iné ditos, Fondo Estanislao Zeballos, Asunción, Editorial Tiempo de Historia, 2015, 346 pp.

a cura di Jacopo BASSI

Estanislao Zeballos è stato un personaggio poliedrico: avvocato, politico, giornalista, storico, geografo ed etnografo, si è occupato di un gran numero di argomenti e si è impegnato in altrettante attività. Questo libro nasce da un'impresa che Zeballos non è mai riuscito a condurre a termine: scrivere una storia della guerra del Paraguay. Malgrado l'intento non sia mai stato raggiunto, Zeballos aveva già raccolto una considerevole mole di materiale. Ed è proprio del recupero di questo complesso documentale che si è occupata la curatrice dell'opera, Liliana Brezzo; lo ha fatto 
attingendo al Fondo Estanislao Zeballos, che è stato devoluto nel 1982 allo Stato del Paraguay (oggi è conservato presso l'Archivio dell'Istituto e Museo di Storia militare).

È proprio da questa prospettiva di recupero che nasce il titolo "La guerra del Paraguay in prima persona"; il libro è in realtà un'analisi utile tanto al recupero di testimonianze inedite sulla Guerra del Paraguay quanto ad indagare i meccanismi attraverso cui operava Zeballos per raccogliere le fonti per scrivere la sua opera. Le testimonianze stesse mostrano una cultura della storia orale ancora acerba (nella maggior parte dei casi non viene indicata la lingua nella quale si esprimeva l'intervistato, se si sia fatto ricorso a un interprete...). L'attento lavoro di Liliana Brezzo, come ammette la stessa curatrice, ha trasformato la raccolta di materiali di Zeballos in un vero testo letterario. 


\section{* Gli autori}

Jacopo Bassi è dottorando dell'Università di Genova. Nel corso dei suoi studi si è occupato di storia della Grecia e dell'Albania in età contemporanea e di storia e istituzioni della Chiesa ortodossa. Lavora per le case editrici Il Mulino e Zanichelli.

URL: < http://www.studistorici.com/2009/02/24/jacopo_bassi/ >

Carla Peñaloza Palma (Santiago de Chile, 1973) es doctora en historia por la Universidad de Barcelona y académica de la Universidad de Chile, lugar donde hizo sus estudios de licenciatura en Historia, y licenciatura en Sociología, y maestría en Historia. Sus principales temas de investigación son Memoria y derechos humanos, en los contextos de dictaduras del cono sur. En 2015 publico el libro El Camino de la memoria: de la represión a la justicia en Chile. 19732013, Santiago de Chile, Editorial Cuarto Propio, 2015.

URL: < http://www.studistorici.com/progett/autori/\#Palma >

Magdalena Schelotto es doctora en Humanidades (Universidad Carlos III de Madrid), licenciada y Master en Humanidades y Master en Gestión y Producción en e-learning. Miembro investigador del Instituto de Cultura y Tecnología de la UC3M miembro externo del Instituto de Estudios Ibéricos y Latinoamericanos de la Universidad Sorbonne-Paris IV, Francia. Ha desarrollado su actividad docente en la Universidad Paris Descartes (2015/2016), en la Universidad Carlos III de Madrid (2012/2014) y en la Universidad Sorbonne Paris IV (2009/2011). Su actividad investigadora se centra en la Historia contemporánea latinoamericana, en particular, el Cono Sur y sus relaciones con España. Utiliza la metodología de análisis del discurso y se interesa por la educación digital del futuro, los nuevos paradigmas pedagógicos y el soporte audiovisual como documento para la Historia Contemporánea.

URL: < http://www.studistorici.com/progett/autori/\#Schelotto >

Ariel Ignacio Slavutsky es doctor en Ciencias Sociales por la Universidad Nacional de Jujuy y desarrolla investigaciones (es Becario posdoctoral del CONICET) en torno a la relación entre cultura y dictadura militar. Ha publicado en las revistas «International Journal for Humanities and Social Sciences», «Cuadernos FHyCS-UNJU»y «Revista de la Escuela de Antropologia de la UNR».

URL: < http://www.studistorici.com/progett/autori/\#Slavutsky > 
Ana Belén Zapata es doctora en Historia por la Universidad Nacional de La Plata (UNLP) y docente regular de la Facultad de Filosofía y Letras en la Universidad de Buenos Aires (UBA) de la materia "Teoría e Historia de la Historiografía". Es becaria posdoctoral del Consejo Nacional de Investigaciones Científicas y Técnicas (CONICET). Ha publicado distintos artículos sobre la temática de la conflictividad obrera en los años '6o y '70 en Argentina y sobre la represión en la última dictadura militar de 1976 en Argentina.

URL: < http://www.studistorici.com/progett/autori/\#Zapata >

\section{Per citare questo articolo:}

BASSI, Jacopo, PEÑALOZA PALMA, Carla, SCHELOTTO, Magdalena, SLAVUTSKY, Ariel, ZAPATA, Belen, «Panoramica: ConoSur 2015», Diacronie. Studi di Storia Contemporanea : La voce del silenzio: intelligence, spionaggio e conflitto nel XX secolo, 29/12/2016,

URL:< http://www.studistorici.com/2016/12/29/cono-sur_numero_28/ >

Diacronie Studi di Storia Contemporanea www.diacronie.it

Risorsa digitale indipendente a carattere storiografico. Uscita trimestrale. redazione.diacronie@hotmail.it

Comitato di redazione: Jacopo Bassi - Luca Bufarale - Antonio César Moreno Cantano - Deborah Paci - Fausto Pietrancosta - Alessandro Salvador - Matteo Tomasoni - Luca Zuccolo

Diritti: gli articoli di Diacronie. Studi di Storia Contemporanea sono pubblicati sotto licenza Creative Commons 3.0. Possono essere riprodotti e modificati a patto di indicare eventuali modifiche dei contenuti, di riconoscere la paternità dell'opera e di condividerla allo stesso modo. La citazione di estratti è comunque sempre autorizzata, nei limiti previsti dalla legge. 